\title{
NURBS curve method Taylor's launch type of interpolation arithmetic
}

\author{
Wan-Jun Zhang ${ }^{1,2,3, a}$, Shan-Ping Gao ${ }^{1, b}$ Xi-Yan Cheng ${ }^{1, c}$ \&Feng Zhang ${ }^{2, d}$ \\ 1 Quanzhou Institute of Information Engineering, 36200, China, \\ 2 School of Mechanical Engineering, Xian Jiao tong University, China741049 \\ 3 Lanzhou Industry and Equipment Co. ,Ltd. , Lanzhou 730050, China \\ agszwj_40@163.com, b358391056@qq.com, ${ }^{\text {c326593363@qq.com, }{ }^{d} 116543048 @ q q . c o m}$
}

\begin{abstract}
Keywords: NURBS curve; velocity planning interpolation algorithm; real-time interpolation;
\end{abstract} simulation.

\begin{abstract}
The algorithm of NURBS interpolation was introduced,it is the key for NURBS curve interpolation that formula of Taylor development of first-order, derivation of two order in the interpolation cycle under the condition of certain interpolation increment only and interpolation speed, by changing the interpolation increments can achieve correction curve of interpolation. This paper introduces a method using DSP motion control card for NURBS curve interpolation methods, this method can not only shorten the interpolation time, also can be controlled by motion control card interrupt timer to adjust interpolation. The last example shows that, the interpolation algorithm not only meet the control requirements, but also meets the requirements of high speed and high precision interpolation.
\end{abstract}

\section{Introduction}

The traditional numerical control machining system uses the discretization of the straight line segment approximation. Caused by the velocity profile curve discontinuity and fluctuation, and generate a lot of data, increase the burden of the CNC system, it is difficult to meet the high speed, high precision machining needs [1]. In order to solve this problem, the processing stability, high speed and high efficiency are improved, the Shpitalni and [2] are calculated according to the first order Taylor expansion approximation to calculate the interpolation points. Yang and other [3] based on the use of two order Taylor expansion approximation to calculate the interpolation point, improve the approximation error. Although the above algorithm can obtain a constant speed, without considering the error control [4]. For NURBS curve interpolation [5-8] curve interpolation algorithm and two step design expansion by Taylor, these documents are about NURBS interpolation curve and surface interpolation parameters recursive, Taylor expansion of one order and two order solution of complicated processing error. This paper mainly introduces a NURBS curve interpolation algorithm of Taylor series expansion, one order and two order derivative for NURBS curve interpolation and Taylor interpolation speed increment, which can be simplified into a derivative interpolation; NURBS curve can be achieved in the real-time interpolation of DSP motion control card, can improve the speed and precision of [9] interpolation operation, saving time interpolation.

In modern CAD/CAM systems, NURBS(Non-Uniform Rational B-Spline) more and more widely has used in the CNC ma chine tools field. It has many character[1-3]: NURBS can give a unified mathematical representation for surfaces and curves, NURBS can change the shape by modifying weight vector and control point ,etc. But, NURBS has a shortcoming: interpolation time bigger and NURBS curve interpolation error are not easy changed. Lanzhou Industry and Equipment Co. Ltd.researchers [4-6] proposed an NURBS algorithms which based on real-time interpolation and adaptive interpolation. Literature [7-9] give some NURBS interpolation algorithms, which makes NC programming complicated and interpolation calculate complicated . Shpitalni et al. [9] derived the same interpolation algorithm by using Taylor's expansion. Houng and Yang [10] were given Cubic spline curve interpolator by using Euler algorithm. Literature [11] research on high-grade CNC machines tools CNC system for B-Spline curve method of High-speed 
real-time interpolation arithmetic.Lo and Chung[12-13] proposed the error interpolation algorithm which error calculations changed by curve chord.

On the basis of the research above, a velocity planning interpolation algorithm based on NURBS curve is presented in this paper. Furthermore,velocity planning interpolation algorithm can meet with NURBS curve interpolation. We can use velocity planning interpolation algorithm that calculate $\left(X_{i}, y_{i} z_{i}\right)$. Simulation results show that the proposed NURBS curve interpolator meet the high-speed and high-accuracy interpolation requirements of CNC systems.

\section{Interpolation algorithm of NURBS curve}

\subsection{Mathematical representation of NURBS curves}

In this paper, NURBS curve is used to represent a parametric of a Improved algorithm adaptive of NURBS curve, and it is introduced first. Supposed $p(u)$ can be represented a Improved algorithm adaptive of NURBS curve. While NURBS [3] are parametrically mathematical definition by the following Eq.(1):

$$
p(u)=\frac{\sum_{i=0}^{n} \omega_{i} d_{i, k}(u)}{\sum_{i=0}^{n} \omega_{i} N_{i, k}(u)}=\sum_{i=0}^{n} p_{i} N_{i, k}(u)
$$

Where $u$ is cubic time a Improved algorithm adaptive of NURBS curve each parameter, $\mathrm{k}$ the order of a Improved algorithm adaptive of NURBS curve . $p_{i}$ is the control points, $\omega_{i}$ is the weight vector , $N_{i, k}(u)$ is the blending function .

$$
N_{i, k}(u)=\left\{\begin{array}{c}
N_{i, k}=\left\{\begin{array}{l}
1, u_{i} \leq u \leq u_{i+1} \\
0, \text { other }
\end{array}\right. \\
N_{i, k}=\frac{u-u_{i}}{u_{i+k+1}-u_{i}} N_{i, k-1}(u) \\
\quad+\frac{u_{i+k+1}-u}{u_{i+k+1}-u_{i+1}} \\
\text { define }: \frac{0}{0}=0
\end{array}\right.
$$

By substituting $U$

$$
U=\{\underbrace{c, \cdots, c}_{p+1}, \boldsymbol{U}_{p+1}, \cdots, \boldsymbol{U}_{m-p-1}, \underbrace{d, \cdots, d}_{p+1}\}
$$

2.2 An algorithm for real time interpolation of NURBS curve Taylor expansion

$$
u_{i+1}=u_{i}+\left.\frac{d u}{d t}\right|_{t=t_{i}} T+\left.\frac{1}{2} \frac{d^{2} u}{d t^{2}}\right|_{t=t_{i}} T^{2}+\text { H.O.T. }
$$

Where, H.O.T. denotes the higher order terms.

1)Parametric equations are expressed by Taylor's formula:

An approximate formula for the first order expansion of Taylor's formula is obtained.

$$
u_{i+1} \approx u_{i}+\frac{V \cdot T}{\left|\frac{d p(u)}{d t}\right|_{U}=u_{i}}
$$

Set NURBS/B curve $p(u)$ is expressed as $T=t_{i+1}-t_{i}$, when the interpolation cycle $T \rightarrow 0$, you can meet $\boldsymbol{u}_{i+1} \approx \boldsymbol{u}_{i}$ the Taylor formula one order expansion interpolation approximate iteration. 


$$
\boldsymbol{u}_{i+1} \approx \boldsymbol{u}_{i}+\frac{V \cdot T}{\left|\frac{d p(u)}{d t}\right| u=u_{i}}-\frac{V^{2} \cdot T^{2}\left(\frac{d u}{d t} \cdot \frac{d^{2} p(u)}{d t^{2}}\right)}{2 \cdot\left|\frac{d p(u)}{d t}\right|}
$$

By the formula (8), we can know that there $R_{i}$ is a radius of curvature of any point.

$$
R_{i}=\frac{1}{\frac{\left|\frac{d p(u)}{d t} \times \frac{d^{2} p(u)}{d t^{2} \mid}\right|}{\left|\frac{p(u)}{d t}\right|^{2}}}=\frac{\left|\frac{p(u)}{d t}\right|^{2}}{\left|\frac{d p(u)}{d t} \times \frac{d^{2} p(u)}{d t^{2}}\right|}
$$

$$
\text { When } \boldsymbol{R}_{i} \rightarrow \infty \text {, Where } \boldsymbol{u}_{i+1} \approx \boldsymbol{u}_{i}+\frac{V \cdot T}{\left|\frac{d p(u)}{d t}\right|_{\boldsymbol{U}}=\boldsymbol{u}_{i}}
$$

If the radius of curvature is small, the Taylor formula satisfies the two order iteration. A derivative of formula (4) and Nikai Misakishikiko (10) it is common type:

$$
\Delta u=u_{i+1}-u_{i}=\frac{V \cdot T}{\left|\frac{d p(u)}{d t}\right|_{u}=u_{i}}
$$

\section{NURBS curve method Taylor's launch type of interpolation arithmetic}

By the formula (10), a recursive approximation is almost equal to the contour error push and the two order approximate recursive calculation, but the two order approximate recursive volume, so this paper uses an order recursive interpolation algorithm.

By using first order recursive interpolation, the first derivative:

$$
\begin{aligned}
& \left\{\begin{array}{l}
x=p_{x}(u) \\
y=p_{y}(u) \\
z=p_{z}(u)
\end{array}\right. \\
& \frac{\mathrm{d} u}{\mathrm{~d} t}=\frac{V}{\left\|\frac{d p(u)}{d t}\right\|}=\sqrt{\left(\frac{d x}{d u}\right)^{2}+\left(\frac{d y}{d u}\right)^{2}+\left(\frac{d z}{d u}\right)^{2}}=\sqrt{x^{\prime 2}+y^{\prime 2}+z^{\prime 2}}
\end{aligned}
$$

Calcuated $x^{\prime}, y^{\prime}, z^{\prime}$

$$
\left\{\begin{array}{l}
x^{\prime}=\frac{d p_{x}(u)}{d u} \\
y^{\prime}=\frac{d p_{y}(u)}{d u} \\
z^{\prime}=\frac{d p_{z}(u)}{d u}
\end{array}\right.
$$

Supposed $\frac{d u}{d t}$, where

$$
\frac{d u}{d t}=\frac{V}{\left|\frac{d p(u)}{d u}\right|}=\frac{V}{\sqrt{x^{\prime 2}+y^{\prime 2}+z^{\prime 2}}}
$$

In CNC system, the interpolation period is always constant as $T=t_{i+1}-t_{i} \square$, then $u_{i+1}$ 


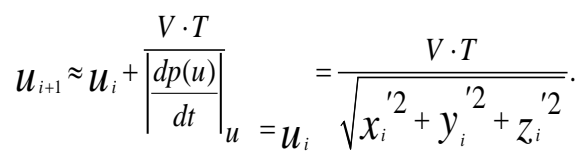

From Eq.(1), Eq.(13) , Eq.(14), Eq.(15)we should get , Eq.(16).

$$
x^{\prime}(u)=\frac{\sum_{i=0}^{n+2} \omega_{i} N_{i, k}(u) \cdot \sum_{i=0}^{n+2} \omega_{i} X_{i} N^{\prime}{ }_{i, k}(u)}{\left[\sum_{i=0}^{n+2} \omega_{i} N_{i, k}(u)\right]^{2}}=-\frac{\sum_{i=0}^{n+2} \omega_{i} N^{\prime}{ }_{i, k}(u) \cdot \sum_{i=0}^{n+2} \omega_{i} X_{i} N_{i, k}(u)}{\left[\sum_{i=0}^{n+2} \omega_{i} N_{i, k}(u)\right]^{2}}
$$

Where,

$$
\begin{gathered}
N_{i, k}^{\prime}(u)=k\left[\frac{N_{i, k-1}(u)}{u_{i+k}-u_{i}}-\frac{N_{i+1, k-1}(u)}{u_{i+k+1}-u_{i+1}}\right] \\
y^{\prime}(u)=\frac{\sum_{i=0}^{n+2} \omega_{i} N_{i, k}(u) \cdot \sum_{i=0}^{n+2} \omega_{i} y_{i} N^{\prime}(u)}{\left[\sum_{i=0}^{n+2} \omega_{i} N_{i, k}(u)\right]^{2}}=-\frac{\sum_{i=0}^{n+2} \omega_{i} N^{\prime}{ }_{i, k}(u) \cdot \sum_{i=0}^{n+2} \omega_{i} y_{i} N_{i, k}(u)}{\left[\sum_{i=0}^{n+2} \omega_{i} N_{i, k}(u)\right]^{2}} \\
z^{\prime}(u)=\frac{\sum_{i=0}^{n+2} \omega_{i} N_{i, k}(u) \cdot \sum_{i=0}^{n+2} \omega_{i} Z_{i} N^{\prime}(u)}{\left[\sum_{i, k}^{n+2} \omega_{i} N_{i, k}(u)\right]^{2}}=-\frac{\sum_{i=0}^{n+2} \omega_{i} N^{\prime}{ }_{i, k}(u) \cdot \sum_{i=0}^{n+2} \omega_{i} Z_{i} N_{i, k}(u)}{\left[\sum_{i=0}^{n+2} \omega_{i} N_{i, k}(u)\right]^{2}}
\end{gathered}
$$

From Eq.(16), Eq.(17), Eq.(19), we should get $\left(x_{i}, y_{i}, Z_{i}\right)$, NURBS curve method Taylor's launch type of interpolation arithmetic should be finished.

\section{NURBS curve method Taylor's launch type of interpolation arithmetic flow chart}

NURBS curve method Taylor's launch type of interpolation arithmetic based on NURBS curve is explained as follow:

Step1: Input date are from NURBS curve parameters model, such as NURBS curve control points, weight vector and so on.

Step2: By using two order of Taylor's expansion is Calcuated $u_{i+1}$.

Step3: Calcuated $x^{\prime}, y^{\prime}, z^{\prime}$.

By using Eq.(7), Eq.(10) and Eq.(13), the NURBS curve position $\left(x_{i}, y_{i} z_{i}\right)$ can be calculated by using $u_{i+1}$. By substituting $u_{i+1}$

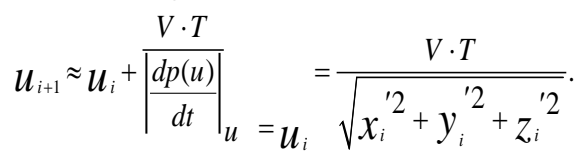

Step4: NURBS curve interpolation is finished. 


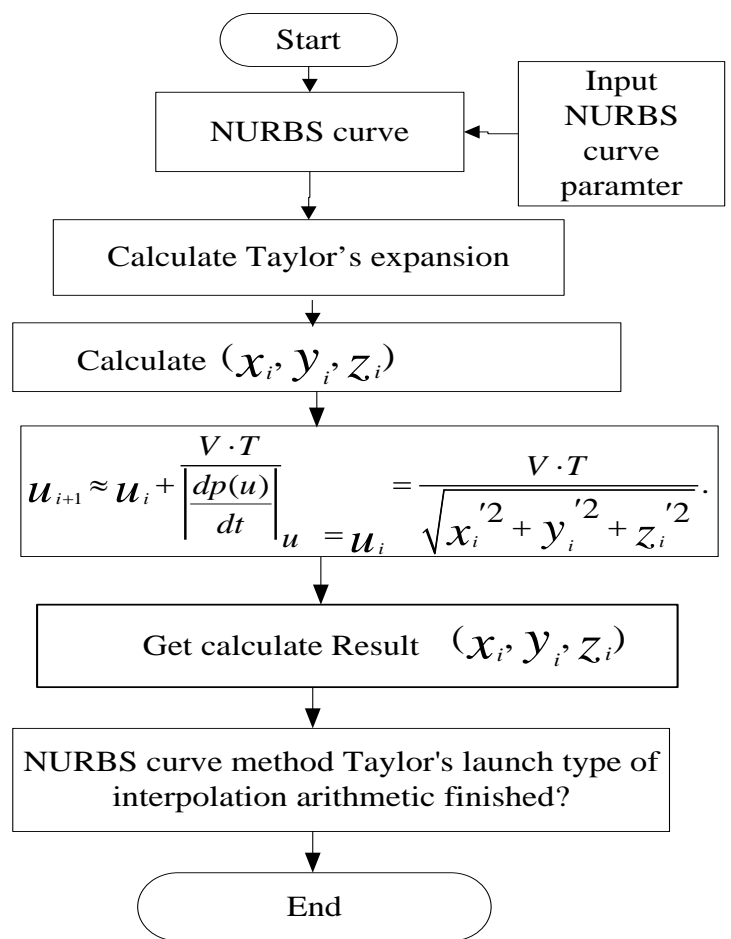

Fig .1: Flowchart of algorithm

\section{Experiment simulation and analysis}

5.1 Interpolation example

In this simulation ,this interpolation scheme is realized on the motion controller developed by our own lab, based on DSP TMS20C543,Development environment is a PC with AMD Sempron $2,800+2.1 \mathrm{Ghz}$ CPU,2GB RAM, and main frequency is $1.44 \mathrm{MHz}$,machine tool is machine center. Machining parameters and dynamics parameters are shown in Table 1.

In the paper, velocity planning interpolation algorithm based on NURBS curve parameter ,the control points, weight vector, and knot vector of NURBS for the provided example are assigned as follows:

$$
p\left(x_{i}, y_{i}, z_{i}\right)=\left\{\begin{array}{llllllll}
1 & 1.1 & 1.3 & 1.5 & 1.5 & 1.6 & 1.7 & 1.8 \\
1 & 1.1 & 1.2 & 1.5 & 1.5 & 1.7 & 1.8 & 1.9 \\
5 & 10 & 13 & 14 & 15 & 16 & 17 & 19
\end{array}\right\}^{T} \quad i=1,2,3, \cdots
$$

Some of the data interpolation calculation, as shown in Tab.1.

Tab. 1 Some of the data interpolation calculation Table

\begin{tabular}{lllllll}
\hline $\mathrm{I}$ & $\boldsymbol{X}_{i}$ & $y_{i}$ & $\boldsymbol{Z}_{i}$ & $\boldsymbol{U}_{i}$ & $\Delta \boldsymbol{U}_{i+1}$ & $\sqrt{\Delta x^{2}+\Delta y^{2}+\Delta \mathrm{z}^{2}}$ \\
\hline 0 & 1.33 & 1.433 & 18.211 & 0.000 & 0.00261 & \\
\hline 1 & 1.34 & 1.5653 & 18.278 & 0.061 & 0.00267 & 0.23365 \\
\hline 2 & 1.345 & 1.6892 & 18.451 & 0.064 & 0.00268 & 0.23364 \\
\hline 3 & 1.3577 & 1.7309 & 18.811 & 0.062 & 0.00265 & 0.23362 \\
\hline
\end{tabular}

The allowable acceleration and jerk $J=3200 \mathrm{~mm} \cdot \mathrm{s}^{-2}$, the maximum value of the chord error $\delta_{\text {max }}=1.5 \mu \mathrm{m}$, the command interpolation federate $F=1 \mathrm{~mm} \cdot \mathrm{s}^{-1}$.

The SIEMENS 810D CNC system has a wide and large share in the CNC system market ,and they make NC parameters as NC program command CNC machine tool, Velocity planning interpolation algorithm based on NURBS curve G code .G06.2: NURBS curve.txt, RT ' in the CNC machine tools complete interpolation.

G06.2 K4 U0 X1.0 Y0 Z0 W1.0

$\mathrm{U} 0 \mathrm{X} 2.0 \mathrm{Y} 1.0 \mathrm{Z} 10 \mathrm{~W} 1 / 5$

$\mathrm{U} 0 \mathrm{X}-1.0 \mathrm{Y} 1.0 \mathrm{Z} 10 \mathrm{~W} 1 / 5$

U0 X-2.0 Y0 Z20 W1

$\mathrm{U} 2$ 
Figures 2 and Figures 3, for CNC machine tool in velocity planning interpolation algorithm based on NURBS curve.

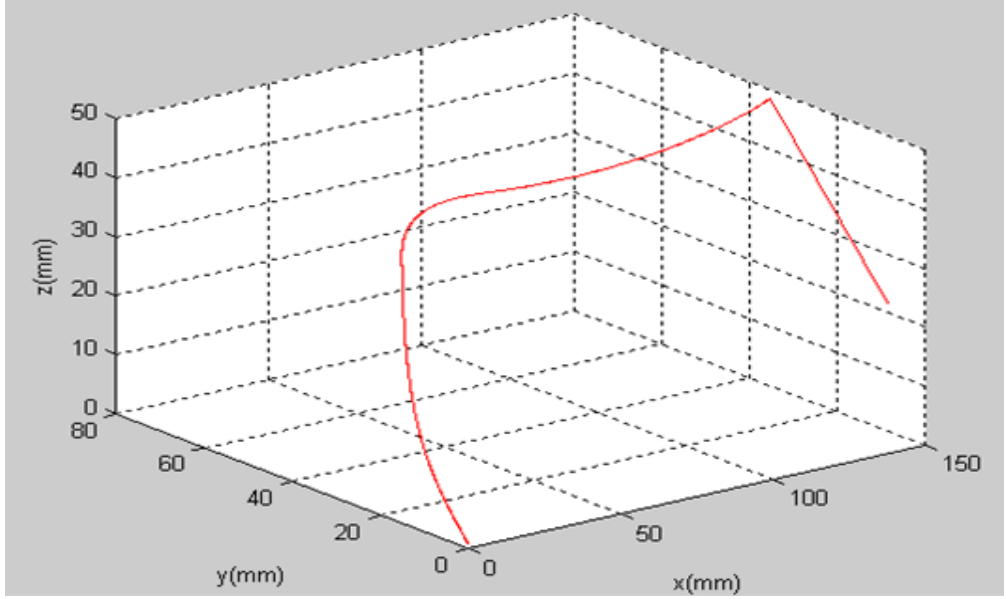

Fig. 2 : Velocity planning interpolation algorithm based on NURBS curve

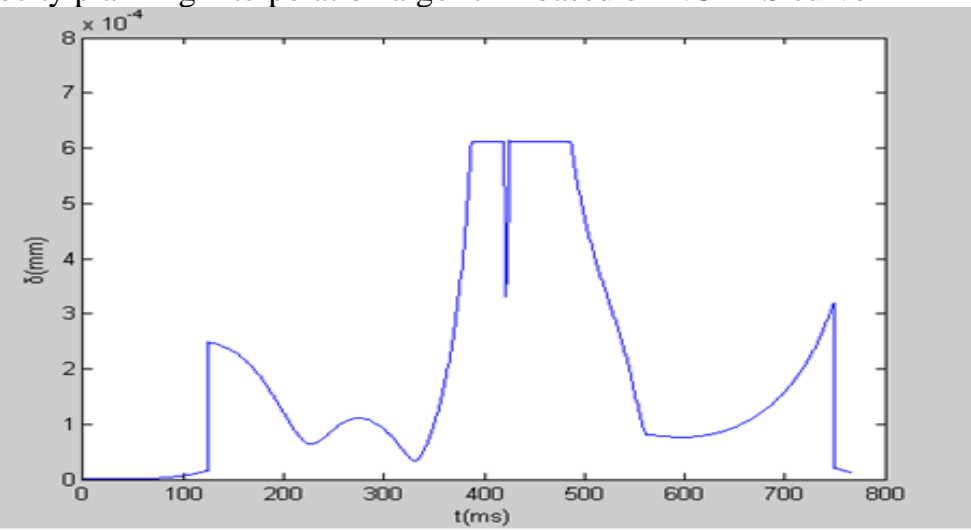

Fig. 3 : Comparison figure of interpolation error

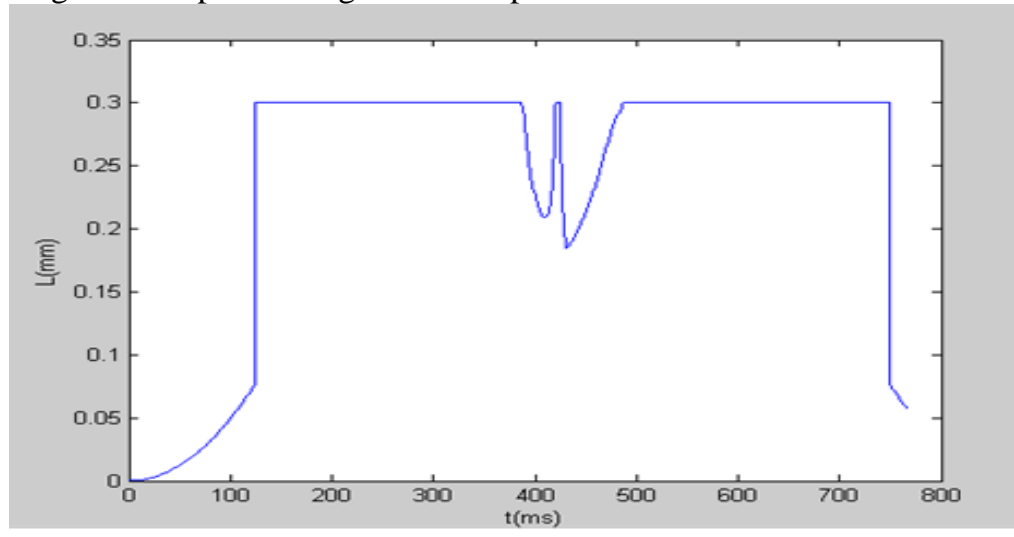

Fig. 4 : Comparison figure of interpolation error

\subsection{Data analysis}

In order to show the superiority of velocity planning interpolation algorithm, the interpolation accuracy and the interpolation time and the interpolation time data table are made according to the graph of Figure3, as shown in Table 2.

Tab.2 Table analysis of interpolation of NURBS curve results

\begin{tabular}{|c|c|c|c|}
\hline Parameters algorithms & $\begin{array}{l}\text { Interpolation } \\
\text { time(s) }\end{array}$ & $\begin{array}{l}\text { Max interpolation } \\
\text { error }(\mathrm{mm})\end{array}$ & $\begin{array}{l}\text { Min interpolation } \\
\text { error }(\mathrm{mm})\end{array}$ \\
\hline $\begin{array}{l}\text { NURBS curve interpolation } \\
\text { algorithm }\end{array}$ & 17.84 & 0.90801 & 0.212 \\
\hline $\begin{array}{l}\text { Velocity planning interpolation } \\
\text { algorithm }\end{array}$ & 15.5 & 0.59801 & 0.046 \\
\hline
\end{tabular}


As can be seen from Fig. 3 and table 2, in the process of the interpolation, interpolation time reduced , Max interpolation error deceased, Minx step error value is 7.980, which meet the expected to velocity planning interpolation algorithm, i.e. to reduce interpolation time and Max interpolation error. To verify the high efficiency and reliability of this velocity planning interpolation algorithm are applied in the experiments to make a comparison .It can be seen that velocity planning interpolation algorithm based on NURBS curve is feasible and efficent.

\section{Summary}

In the paper, velocity planning interpolation algorithm based on NURBS curve is introduced. Firstly, the second-order Taylor expansion is applied on the numerator in NURBS curve representation with parameter curve. Then,velocity planning interpolation algorithm can meet with NURBS curve interpolation .We can use velocity planning interpolation algorithm that calculate $\left(\boldsymbol{x}_{i}, \boldsymbol{y}_{i}, \boldsymbol{z}_{i}\right)$. Simulation results show that the proposed NURBS curve interpolator meet the high-speed and highaccuracy interpolation requirements of CNC systems. The interpolation of NURBS curve should be finished. In addition,NC machining time can be reduced. Implementation on NC machine has proven the feasibility of a developed interpolation algorithm. The simulation results show that the algorithm is correct; it is consistent with a NURBS curve interpolation requirements.

\section{Acknowledgements}

The authors thank the financial supports from National Natural Science Foundation of China(Grant no. 51165024) and Science and Technology Major Project of "High-grade NC Machine Tools and Basic Manufacturing Equipment” (2010ZX040001-181).

\section{References}

[1] Fang Yi-Xiang, Liu Wen-Xue. Based on the geometric properties of the cubic uniform B-Spline curve structure description[J]. Journal of engineering graphics, 2 , pp.96-102, 2006.

[2] Zhang Wan-Jun, Hu Chi-Bing, Zhang Feng ,et al . Honing machine motion control card three B spline curve method of interpolation arithmetic for CNC system [J].Chinese Journal of Manufacturing Technology \& Machine Tool , 8(8), pp.40-43,August 2012.

[3] Zhang Wan-Jun, HU Chi-bing, WU Zai-xin, et,al. Research on modification algorithm of Three B Spline curve interpolation technology [J].Chinese Journal of Manufacturing Technology \& Machine Tool ,2 pp.141-143,Feburary 2013.

[4] Zhang Wan-Jun, Zhang Feng, Zhang Guo-hua. Research on a algorithm of adaptive interpolation for NURBS curve. [J].Applied Mechanics and Materials ,Vol. 687-691, pp.16001603, December 2014.

[5] Zhang Wan-Jun, Zhang Feng, Zhang Guo-hua. Research on modification algorithm of Cubic Bspline curve interpolation technology. [J].Applied Mechanics and Materials, Vol. 687-691, pp.1596-1599, December 2014.

[6] Zhang Wan-Jun, Zhang Feng, Zhang Wan-Liang. Research on a NURBS curve of timing / interrupt interpolation algorithm for CNC system [J].Chinese Journal of Manufacturing Technology \& Machine Tool , 4(4), pp.183-187,April 2015.

[7] Kong Fan-Guo, Hao Shang-Hua,ZhongYan-Zhi. NURBS curve interpolation algorithm to achieve the VC [J]. China new technology and new products, 17, pp.8-9, 2009.

[8] Ye Bo-Sheng,Yang Shu-Zi. CNC system in cubic B-Spline curve interpolation method [J ]. China Mechanical Engineering, 9 ( 3) , pp. 42 - 43, 1998.

[9] Li He-Cheng, Wang Yu-Ping, An interpolation based genetic algorithm for sloving nonlinear bilevel programming problems.Chinese Journal of Computers, 31(6), pp.910-918, June 2008.

[10] Shpitalni M, Koren Y, Lo CC. Realtime curve interpolators. Computer-Aided Design, 26, pp.832-838, 1994. 
[11] Zhang Wan-Jun, Zhang Feng, Zhang Wan-liang. Research on high-grade CNC machines tools CNC system for B-Spline curve method of High-speed real-time interpolation arithmetic [J]. Chinese Journal of Manufacturing Technology \& Machine Tool , 8(8), pp.172-176,August 2015.

[12] Huang JT,Yang DCH.A generalized interpolator for command generation of parametric curves in computer controlled machines. Japan/USA Symposium on Flexible Automation,1(1),pp.393399,January1992.

[13] Lo CC,Chung CY.Curve generation and control for biaxial machine tools. J.CSME ,18, pp.175-182,1997.

[15] Zhang wanjun.A Newton iteration-Rapson of NC interpolation system [P]. China : 201520043082.8,2015.

[16] Zhang wanjun. Based on b-spline curve real-time interpolation of CNC system at a high speed [P]. China : 201520069002.6,2015.

[17] Zhang wanjun. A kind of based on ARM + FPGA dual CPU of NC interpolation system [P]. China : 201520969163. 0,2016.

[18] Zhang wanjun. A control system of numerical control for interpolation PCI system [P]. China : 201620110057.1,2016.

[19] Sun, Shujie, Lin, Hu; Zheng, Liaomo. A real-time and look-ahead interpolation methodology with dynamic B-spline transition scheme for CNC machining of short line segments [J ]. Internation journal of advanced manufacturing technology, 84 ( 3) , pp. 1359-1370, 2016.

[20] Duan, Molong, Okwudire, Chinedum. Minimum-time cornering for CNC machines using an optimal control method with NURBSparameterization

[J]. Internation journal of advanced manufacturing technology, 85 ( 3) , pp. 1405-1418, 2016.

[21]Liu Qiang,Liu Huan,Yuan Songmei.High Accurate Interpolation of NURBS Tool Path for CNC Machine Tools[J]. China journal of mechanical engineering, 29 ( 5) , pp. 911-920, 2016.

[22] Zhang Liqiang,Zhang, Kai Yan, Yecui.Local Corner Smoothing Transition Algorithm Based on Double Cubic NURBS for Five-axis Linear Tool Path[J].Strojniskivestnik-journal of mechanical engineering, 29 ( 5) , pp. 647-656 , 2016(9). 